\title{
Repetitive Peripheral Magnetic Stimulation in Stroke Rehabilitation - A Case Study
}

\author{
Lavinia Grozoiu, Savulescu Simona, Stefan Hesse, Adrian Bighea, and Mihai Berteanu
}

\begin{abstract}
Treatment of post stroke spasticity represents a major challenge in neurologic rehabilitation. Conventional medication treatment is not enough to control the negative effects of muscle stiffness, lack of movement coordination and control, and consecutive limited range of joint motion especially on affected chronic patients where the immobility-related changes of the mechanical muscle properties are prevailing. Repetitive peripheral magnetic stimulation (rPMS) of affected muscles administered during stretching is a new and attractive option of therapeutical intervention, able to reduce pathological muscle tone and to stimulate cortical reorganization.
\end{abstract}

Index Terms-Muscle stiffness, peripheral repetitive magnetic stimulation, stroke rehabilitation.

\section{INTRODUCTION}

In the whole world, stroke is considered to be a major health problem. Demographic analysis for European Union countries showed that during the last decades population over 65 is in continuous numerical increase, with a higher weight for the age group of 80 years and older. Population aging is a general phenomenon for European countries. The increase of the elder population dependency ratio is able to cause concern [1]. Chronic diseases, especially stroke, frequently encountered in elderly patients, contribute to functional disability, lower quality of life and increase long-term care costs and overloads health services. All the stroke survivors require rehabilitation in order to help them to become as independent as possible and to attain the best possible quality of life. Generally, stroke can cause five types of impairments: paralysis or lack of control concerning movement, sensitive disturbances and pain, problems in using or understanding language, problems with thinking and memory, and emotional disturbances. Patients with post stroke motor deficits, often require the use of an assistive device such as canes, wheelchair, walkers and robotics. Walking aids

Manuscript received April 21, 2015; revised June 5, 2015. This work was supported by the Sectorial Operational Program Human Resources Development (SOP HRD), financed from the European Social Fund and by the Romanian Government under the contract number POSDRU/159/1.5/S/137390. This work was supported by a grant of the Romanian National Authority for Scientific Research, CNDI- UEFISCDI, project number 190/2012, as well as by COST Action TD1006 European Network on Robotics for NeuroRehabilitation.

L. Grozoiu, S. Savulescu, and M. Berteanu are with the Rehabilitation Department of Elias University Hospital, Carol Davila University of Medicine and Pharmacy of Bucharest, Romania (e-mail: laviniagrozoiu@gmail.com, simona.manoila@yahoo.com, mberteanu@gmail.com).

S. Hesse is with Medical Park Berlin Neurological Rehabilitation, Charite University of Medicine, Berlin, Germany.

A. Bighea is with University of Medicine and Pharmacy, Craiova, Romania. functions are to increase stability and to improve muscle tension. Canes and walkers appear to be effective in compensating for patients' decreased postural sway and for enhancing their participation in activities of daily living [2], especially if they have hemiplegia [3], [4]. But, the most important rehabilitation intervention approach must be the restorative one, not the assistive one. In order to regain functionality, the stroke survivor has to undergo a complex program of training (physical exercises aided or not by advanced rehabilitation technology) and different physical agents applications, aimed to restore the optimal condition of the nervous and myo-arthrokinetic structures involved in functional movement [5].

The upper and lower limb muscle stiffness and consecutive limited range of joint motion after stroke can seriously interfere with daily living activities, and has an important influence in quality of life. Three months after stroke for instance, $25 \%$ of the surviving patients present an upper limb and $15 \%$ of them - lower limb stiffness [6], [7].

Spasticity being an important cause of disability, the treatment of this complication represents a major challenge for neurological rehabilitation.

Conventional treatment of muscle stiffness, like passive mobilization, physical therapy, oral antispastic medication, serial casting, surgery and the intrathekal application of Baclofen, seems to be non-effective. Clinical trials showed that only Botulinum toxin (BTX) injection is an efficient therapy for muscle stiffness, but with limited effectiveness in severely affected chronic patients where the immobility-related changes of the mechanical muscle properties are prevailing. Furthermore, the BTX-treatment is expensive. Conventional electrical stimulation is not appropriate for being applied in spasticity, due to the specific condition of the skin of these patients, and do not give good results in spasticity (central neurological issue) as it does in the case of muscle stiffness associated with peripheral nerve-muscle-joint-bone acute or chronic conditions.

Repetitive magnetic stimulation of brain, skeletal muscles, peripheral nerves, or spinal roots, is a non-contact, non-invasive and painless method that can be an alternative in the management of muscle stiffness [8]. More than this, the magnetic stimulation device is easy to use and simple to apply. The painless and non-invasive repetitive peripheral magnetic stimulation (rPMS) has been successfully applied in neurologically impaired adults and children with cerebral palsy $(\mathrm{CP})$ in order to reduce muscle tone improve motor function and perceptual cognitive tasks.

Struppler et al. reported positive effects of rPMS on upper limb spasticity and motor recovery in chronic stroke subjects and associate the improvements on muscle tone and motor control with a significant increase of neural activation $[5,6]$. 
Flamand et al. applied multiple sessions of rPMS of the tibial and common peroneal nerve in CP children, which effected a sustained reduction of lower limb spasticity and improved motor control [9]. Krewer at al. and Struppel et al. applied 20 min of rPMS twice a work day, for two weeks, to patients with spasticity following stroke and they observed relevant effects on muscle tone only for those patients with modified Ashworth Scale scores between 3 - 5 [10].

Other authors noticed clinical reduction of muscle tone at both ankles on children with cerebral palsy after they performed theta-burst stimulation with 900 pulses per minute [9]. It seems that rpMS decrease muscle stiffness and reduce sensitivity of the muscle spindles. Hagbarth et al. studied the thixotrophic phenomenon in healthy subjects [11]. To be published studies of Hesse et al. show that a single session of verum rPMS, in combination with manual muscle stretch,effects a significantly larger decrease of the joint extension deficit in chronic patients with severe spasticity, when compared to sham stimulation.

\section{OBJECTIVE OF THE STUDY}

This study's objective was to test whether rPMS, while stretching supra-maximal the target joints and accompanied by a routine rehabilitation, could lead to a reduction of muscle stiffness on a patient with SNC lesions.

\section{METHOD}

This study investigate two patients with severe and moderate-muscle stiffness, after stroke lesions.

Patient's characteristics:

- The target joints of the upper and/or lower limb did not reach the neutral position when passively moved, even when applying a strong force. The corresponding value on the modified Ashworth score (0-5) was 3 or 4, i.e. the joint could be moved minimally, but was not fixed.

- A conventional X-ray excluded an osseous joint contracture and/or a myositis ossificans.

- A preceding Botulinum A toxin injection with a dosage according to national guidelines had not resulted in a relevant stiffness reduction. The time interval since the last injection was at least four months.

- Any metal implants, deep vein thrombosis, open wounds and relevant edema in the extremities under investigation as well as pacemaker excluded the patient.

- Patient understood and signed the written informed consent of the study, which was approved by the local ethical committee.

Four target joints were studied, in order to assess knee flexor stiffness and plantar flexor stiffness.

\section{EXPERIMENTAL DESIGN}

Initially the patient participated in a familiarization session, in order to be introduced with the study algorithm, the measurement protocol and the mechanism of the rPMS. Three investigators, two therapists and one MD, were responsible.

A Magstim Rapid stimulator with two circular coils (diameter 8 inches) delivered the rPMS. The coil was placed over the muscles corresponding to the target joint, the hamstrings and the plantar flexor group (in case of knee flexor stiffness) and the plantar flexor group (in case of plantar flexor stiffness). During the stimulation period, one therapist moved the target joint in the most outstretched position without eliciting pain. The coil was placed with the A-side on the limb and moved it parallel to the skin covering the targeted muscles.

The patients received 10 sessions (1 time per day, ten days consecutive) of low-frequency rPMS on the affected muscle while the target joint was continuously stretched.

The stimulation parameters were: $60 \%$ intensity, $5 \mathrm{~Hz}, 3 \mathrm{~s}$ trains of stimulation and 3 s pause, a total of 750 stimuli per targeted muscle group. The intensity was above the motor threshold. These stimulation parameters have been used in previous studies [12].

\section{OUtCOME MeAsures}

A blinded investigator assessed the patient while lying supine; the joint under investigation was positioned on a supportive pillow, after the patient had been instructed to extend the joint to their individual maximum. Given the poor interpretation reliability of the goniometer measurements, the same investigator made all the measurements all along the study.

Dependent variable were the relative active extension deficit and Modified Ashworth Scale of the muscles, assessed at baseline (when the patient received only physiotherapy), daily at a fixed time before and after stimulation, and 10 days later (T10).

All outcome measures were assessed 13 times: pre-intervention baseline assessments were repeated 5 days apart (T-10, T-5, T0), T1-direct after the $1^{\text {st }}$ session, T2- 24 hours after the $1^{\text {st }}$ session, $\mathrm{T} 3$ - direct after $2^{\text {nd }}$ session, T4, $\mathrm{T} 5$ - before and after $5^{\text {th }}$ session, T6,T7 - before and after $9^{\text {th }}$ session, T8, T9 - before and after $10^{\text {th }}$ session.

At each measurement point, the investigator assessed the extension deficit with the help of a goniometer at rest. Every assessment was followed by the joint repositioning on the supportive pillow, with no intervention.

\section{Statistics}

As a first step, the extension deficit of each joint relative to the maximum anatomical joint flexion was calculated. The corresponding degrees of the lower limb joints were $150^{\circ}$ (knee joint) and $30^{\circ}$ (ankle plantar flexion). The relative extension deficit and the modified Ashworth score were calculated with the help of a computerized statistical program (SPSS 17.0).

\section{RESULTS}

The patients completed the study with no adverse events from either aspect of intervention. During the baseline (time period T-10 to T0) the active extension deficit at rest did not decrease significantly (only with $5.76 \%$ ). Immediately 
following the first verum stimulation, the mean extension deficit at rest decreased significantly (with $21.11 \%$ ). The positive effects following verum stimulation were fully sustained 3 hours later, at 24 hours the effects had waned but not yet reached the baseline values. A significantly larger decrease from baseline to T9 (of 75.65\%) was observed (see Fig. 1).

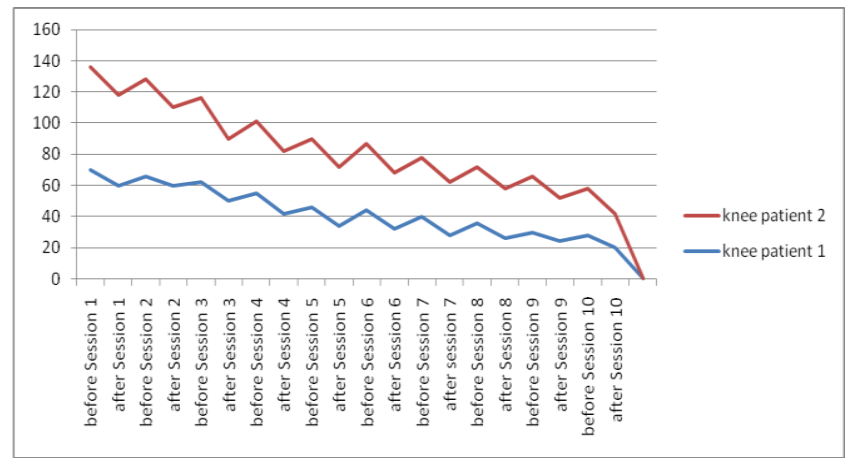

Fig. 1. Graphical representation of progress of the extension deficit for knee joint during intervention period. The measurements are performed before and after each rPMS session.

The mean modified Ashworth scores showed a similar behavior, i.e. no significant change during the baseline, followed by a significant reduction after the verum stimulation. Muscle tone was reduced within the 10 days of stimulation with 1.25 points on the Ashworth score.

\section{DISCUSSION}

A single session of verum rPMS in combination with continuous joint stretch diminished significantly the extension deficit of the stiff joints.

The patients were in the chronic stage, i.e. spasticity in the sense of an altered muscle activity level and immobility-related muscle contractures with sarcopenia, both contributed to the clinical picture of inherent and reflex mediated muscle stiffness and limited range of joint mobility.

The chosen protocol of an exclusive stimulation of the flexor muscles at a frequency of $5 \mathrm{~Hz}$ and 600 stimuli per targeted muscle in combination with continuous muscle stretch followed the work of Zschorlich et al. in healthy subjects, they had noticed a reduced muscle tone and a diminished stretch reflex [12].

The degree of spasticity at baseline seems to be a major predictor of the response to the rpMS intervention, irrespective of the stimulation protocol. Struppler et al. had studied patients with spasticity values by means of the modified Ashworth Scale between 3 and 5. In patients with initial modified Ashworth scores of 1 or 2 however, neither Krewer et al. nor Müller et al. found a relevant effect on muscle tone (both followed the Struppler protocol).

Muscle stretch itself reduced muscle stiffness and many recent studies showed that rPMS itself decrease muscle stiffness. Starting from this our further studies try to see if is a connection between different characteristics of the patient (i.e type of SNC lesion, age of the patients, number of the session of rPMs, different frequency of rPMS) and response of stimulation. In this case we observed positive effects after one single session of rPMS that were maintained after 24 hour, waned but not reached the baseline values. More important after every session the positive effect sum, so at the end of the 10 consecutives workdays sessions it was a significant decrease of muscle stiffness.

\section{CONCLUSION}

For patients with residual spasticity after stroke repetitive peripheral magnetic stimulation is a safe and feasible approach which appears to provide positive effects, with no clear long term benefits.

Further studies with rPMS in larger and randomized population are required, to elucidate if combining rPMS while stretching is better alternative than rPMS without stretching in treatment of muscle stiffness is our next aim as well as to try to determinate the right parameters for patients depending on the characteristics of each.

\section{ACKNOWLEDGMENT}

This paper is supported by the Sectoral Operational Programme Human Resources Development (SOP HRD), financed from the European Social Fund and by the Romanian Government under the contract number POSDRU/159/1.5/S/137390. This work was supported by a grant of the Romanian National Authority for Scientific Research, CNDI- UEFISCDI, project number 190/2012, as well as by COST Action TD1006 European Network on Robotics for NeuroRehabilitation.

\section{REFERENCES}

[1] The World Health Report, 2003.

[2] G. R. Fernie and P. J. Holliday, "Volume fluctuations in the residual Limbs of lower limb amputees," Arch Phys Med Rehabil, vol. 63, no. 4, pp. 162-165, 1982

[3] T. Yuasa, A. Maeda, and S. Y. Higuchi, "Quantitative EEG data and comprehensive ADL (activities of daily living) evaluation of stroke survivors residing in the community," Jounal Physiol. Anthropol. Appl. Human Science, vol. 20, no. 1, pp. 37-41, 2001.

[4] B. Isaacs, D. May, and U. S. Nayak, "The life-space diary: A measure of mobility in old people at home," Intl. Rehabil. Med, vol. 7, no. 4, pp. 182-186, 1985.

[5] A. Struppler, P. Havel, and P. M. Barna, "Facilitation of skilled finger movements by repetitive peripheral magnetic stimulation (RPMS)-A new approach in central paresis," Neuro Rehabilitation, vol. 18, pp. 69-82, 2003.

[6] A. Struppler, B. Angerer, C. Gundisch, and P. Havel, "Modulatory effect of repetitive peripheral magnetic stimulation on skeletal muscle tone in healthy subjects: Stabilization of the elbow joint," Experimental Brain Research, vol. 157, pp. 59-66, 2004

[7] J. Wissel, M. Verrier, D. M. Simpson, D. Charles, P. Guinto, S Papapetropoulos, and K. S. Sunnerhagen, "Post-stroke spasticity: Predictors of early development and considerations for therapeutic intervention," National Library of Medicine National Institutes of Health, vol. 7, no. 1, 2014.

[8] S. Hesse and K. H. Mauritz, "Management of spasticity," Curr. Opin. Neurol, vol. 10, no. 6, pp. 498-501, 1997.

[9] V. H. Flammand, L. D. Beaulieu, L. Nadeau, and C. Schneider, "Peripheral magnetic stimulation to decrease spasticity in cerebral palsy," Pediatr. Neurol, vol. 47, pp. 345-348, 2012.

[10] C. Krewer, S. Hartl, F. Müller, and E. Koenig, "The effects of peripheral magnetic stimulation on upper-limb spasticity and impairment in patients with spastic hemiparesis: A randomized, double-blind sham controlled study," Arch Phys Med Rehabil, vol. 95, pp. 1039-1047, 2014.

[11] K. E. Hagbarth, M. Nordin, and L. G. Bongiovanni, "After-effects on stiffness and stretch reflexes of human finger flexor muscles attributed to muscle thixotropy," Journal of Physiology, vol. 482, pp. 215-223, 1995. 
[12] M. Behrens, A. M. Möller, V. Zschorlich, and S. Bruhn, "Repetitive peripheral magnetic stimulation (15 Hz RPMS) of the human soleus muscle did not affect spinal excitability," Journal Sports Science Medicine, vol. 10, no. 1, pp. 39-44, 2011.

Lavinia Grozoiu was born in Moreni, Romania in 1984 and She obtains her bachelor degree in general medicine from the Transylvania University of Medicine, Brasov, Romania, in 2008. After her residency training in the Department of Rehabilitation Medicine of Elias University Hospital Bucharest until September 2014, she conducted an 8 month internship in Berlin, Germany. She is a Ph.D student since 2011 and currently pursuing her study for Ph.D degree in medical rehabilitation, at Carol Davila University of Medicine and Pharmacy Bucharest in collaboration with Medical Park Berlin Humboldtmühle, Charite - University Medicine Berlin. Her research interests include stroke rehabilitation and magnetic field therapy. Dr. Lavinia Grozoiu is an active member of Romanian Society of Rehabilitation.

Simona Savulescu was born in Tirgu-Jiu in 1981. She is absolved in 2007 U.M.F "Carol Davila" Bucharest, Romania, then made the residency until 2012 in the Department of Rehabilitation Medicine of Elias University Hospital and since 2011 is a Ph.D student in medical science at U.M.F Carol Davila Bucharest, Romania. She is a consultant physician since 2012 in the Rehabilitation Medicine Department of Elias University Hospital, Bucharest, Romania since 2012. She is also the electro physiologist of the department with a high interest in magnetic field therapy Dr. Simona Savulescu is an active member of Romanian Society of Rehabilitation, International Society of Physical and Rehabilitation Medicine and of Romanian Society of Electro diagnostic Neurophysiology.

Stefan Hesse comes from the Black Forest. He studied in Freiburg and Munich. After his residency training at the Technical University of Munich, he made primarily through the Development of new therapies for the treatment of patients after stroking a name, including the locomotion, the gai trainer and the arm trainer Bi Manu Track. He is the director of the Stroke Center Berlin and as a professor of Neurorehabilitation at the Charité. Prof. Dr. med Stefan Hesse is a member of the board of the German Society for Neurological Rehabilitation and has numerous publications in international journals rehabilitation especially in the field of stroke rehabilitation:

Mihai Berteanu was born in Bucharest, Romania. He obtains his bachelor degree in general medicine from the Carol Davila University of Medicine and Pharmacy, Bucharest, in 1987. After his residency training in Romania he performed multiple internships abroad (Bruxelles 1997, Berlin 1998 , Washington 2000, Winterthur 2004, Innsbruck 2006). He completed his $\mathrm{Ph} . \mathrm{D}$ degree in field of rehabilitation using electromyography biofeedback, in 2013. He is the chef of the Department of Rehabilitation Medicine at the Elias University Hospital and a professor of Rehabilitation at the Caro Davila University. Prof. Dr. med Mihai Berteanu is an active member of the European Board and AAPM\&R since 2005 and has numerous publications in international journals. 